\title{
Seleção varietal de Phaseolus vulgaris quanto à tolerância ao estresse salino com base em variáveis de crescimento ${ }^{1}$
}

\author{
Cícero Antônio de Souza Araújo², Hugo Alberto Ruiz³, José Cambraia ${ }^{4}$, Júlio César Lima Neves 5 , \\ Maria Betânia Galvão dos Santos Freire ${ }^{6}$, Fernando José Freire ${ }^{6}$
}

\begin{abstract}
RESUMO
Neste estudo objetivou-se avaliar e selecionar variedades de feijoeiro (Phaseolus vulgaris L.) quanto à tolerância ao estresse salino e identificar, usando variedades de feijoeiro com diferentes graus de tolerância, variáveis que auxiliem na discriminação de variedades de feijoeiro quanto à tolerância a esse tipo de estresse, independentemente do mecanismo apresentado pela planta. Os experimentos foram realizados em casa de vegetação. Inicialmente foram avaliadas 48 variedades de $P$. vulgaris (alocadas nas subparcelas) em dois níveis de salinidade (distribuídos nas parcelas): solução nutritiva normal (SNN) a $0,81 \mathrm{dS} \mathrm{m}^{-1}$ e a teste (SNT) a 5,6 dS m m $^{-1}$, obtida pela adição de $\mathrm{NaCl}$ no delineamento experimental em blocos casualizados, com quatro repetições. A partir da massa seca da parte aérea calculou-se a relação percentual de crescimento alcançada na SNT relativo à SNN das variedades, que variou de 138,7 a 54,1\%, discriminando-as de acordo com o critério de Scott-Knott em duas populações: uma com 14 variedades mais "tolerantes" e outra com 34 variedades, onde ficaram agrupadas variedades "moderadamente tolerantes" e "sensíveis". Para identificar variáveis de crescimento que permitam selecionar feijoeiros quanto à tolerância ao estresse salino duas variedades tolerantes (Vermelho e CNF 5574), uma medianamente tolerante (FT 83-86) e uma sensível (LM 30074), classificadas no experimento anterior, foram cultivadas em solução nutritiva com cinco níveis de salinidade $\left(0,81 ; 2,7 ; 4,6 ; 6,5 ;\right.$ e $\left.8,4 \mathrm{dS} \mathrm{m}^{-1}\right)$. Analisando-se a massa seca da raiz, do caule, do pecíolo, das folhas e da parte aérea, a área foliar e a área foliar específica, concluiu-se que a área foliar específica foi o índice que efetivamente mais contribuiu para a discriminação das variedades de feijoeiro quanto à tolerância à salinidade.
\end{abstract}

Palavras-chave: Tolerância a sais, feijoeiro, $\mathrm{NaCl}$, salinidade

\section{ABSTRACT}

\section{Selection of Phaseolus vulgaris L. varieties for tolerance to salt stress based on growth variables}

This study aimed to evaluate and select varieties of bean (Phaseolus vulgaris L.) for tolerance to salt stress and to identify, using bean varieties with different degrees of tolerance, variables to aid in the screening of salt-tolerant varieties, regardless of the mechanism used by the plant. The experiments were conducted in a greenhouse. Initially, 48 $P$. vulgaris varieties were evaluated (allocated in subplots) at two salinity levels (distributed in the plots): normal nutrient solution (NNS) at $0.81 \mathrm{dS} \mathrm{m}^{-1}$ and test solution (TNS) at $5.6 \mathrm{dS} \mathrm{m}^{-1}$, which was obtained by adding $\mathrm{NaCl}$ in a randomized block design with four replications. The dry weight of aerial part was used to calculate the percentage of

Recebido para publicação em novembro de 2008 e aprovado em setembro de 2009

'Parte da Dissertação de Mestrado do primeiro autor apresentada ao Programa de Pós-Graduação em Solos e Nutrição de Plantas da UFV.

${ }^{2}$ Engenheiro-Agrônomo, Doutor. Instituto Federal de Educação Ciência e Tecnologia do Sertão Pernambucano, Rodovia BR 235, km 22, 56.314-520, Petrolina, Pernambuco, Brasil. cicero@cefetpet.br

${ }^{3}$ Bioquímico, Doutor. Departamento de Solos, Universidade Federal de Viçosa, Av. Peter Henry Rolfs, s/n, 36.570-000, Viçosa, Minas Gerais, Brasil. hruiz@ufv.br

${ }^{4}$ Engenheiro-Agrônomo, Ph.Doctor. Departamento de \Biologia Vegetal, Universidade Federal de Viçosa, Av. Peter Henry Rolfs, s/n, 36.570-000, Viçosa, Minas Gerais, Brasil. cambraia@ufv.br

${ }^{5}$ Engenheiro-Agrônomo, Doutor. Departamento de Solos, Universidade Federal de Viçosa, Av. Peter Henry Rolfs, s/n, 36.570-000, Viçosa, Minas Gerais, Brasil. julio.neves@pesquisador.cnpq.br

${ }^{6}$ Engenheiros-Agrônomos, Doutores. Universidade Federal Rural de Pernambuco, Departamento de Agronomia, Rua Dom Manoel de Medeiros, s/n, 52.171-900, Recife, Pernambuco, Brasil. betania@depa.ufrpe.br, f.freire@depa.ufrpe.br 
growth of the varieties achieved in TNS in relation to NNS, which ranged from 138.7 to $54.1 \%$, discriminating them according to the Scott-Knott criterium in two populations: one with 14 more "tolerant" varieties and another with 34 varieties, which grouped "moderately tolerant" and "sensitive" varieties. To identify growth variables that allow selection of tolerant plants to salt stress, two tolerant (Vermelho and CNF 5574), a moderately tolerant (FT 83-86) and a sensitive (LM 30074) varieties, which were classified in a previous experiment, were grown in nutrient solutions with five levels of salinity $\left(0.81,2.7,4.6,6.5\right.$, and $\left.8.4 \mathrm{dS} \mathrm{m}^{-1}\right)$. Results of dry mass of root, stem, petiole, leaves and aerial part, leaf area and specific leaf area showed that the specific leaf area was the index that most effectively contributed to the discrimination of bean varieties for tolerance to salinity.

Key words: Salt tolerance, bean, $\mathrm{NaCl}$, salinity

\section{INTRODUÇÃO}

É reconhecida a existência de diferenças genéticas na tolerância a sais entre espécies e variedades dentro de determinada espécie, Essas diferenças podem ser usadas para identificar plantas mais tolerantes ao estresse salino.

O método convencional de multiplicação de plantas, segundo Epstein \& Rains (1987), tem sido a seleção e o melhoramento de variedades para alta produtividade, resistência a doenças, alta qualidade e outras características desejáveis. A maior parte dos melhoristas têm conduzido essa seleção e melhoramento em condições ambientais favoráveis, pois condições estressantes podem confundir os resultados do melhoramento para as características desejadas. A seleção e melhoramento por meios nãoconvencionais, como cultura de tecidos e de células, biologia molecular, hibridação somática etc., podem apresentar melhores perspectivas de sucesso no desenvolvimento de variedades tolerantes à salinidade, porém, pouco tem sido feito até o momento nessa linha.

A falta de material selecionado e melhorado quanto à tolerância a sais, principalmente quando se trata de grandes culturas, impede o avanço no conhecimento sobre o estresse salino em áreas afins como: fertilidade, física de solo, nutrição de plantas, fisiologia vegetal, fitotecnia etc. Uma alternativa disponível, de imediato, é a seleção das espécies aproveitando suas variabilidades genotípicas quanto à tolerância à salinidade, o que é feito, na maioria das vezes, avaliando-se a redução da produção de plantas crescidas sob estresse salino em relação à produção daquelas crescidas em condições não-salinas (Allison, 1964; Fageria, 1985; Araújo et al., 1992). As variáveis mais utilizadas em estudos dessa natureza, com várias culturas, são a produção de massa seca da raiz, da parte aérea e a total, bem como a área foliar, avaliadas em trigo (Mahmood \& Quarric, 1993), pepino (Chartzoulakis, 1991), melão (Franco et al., 1993; Silva et al., 2005), tomate (Sanchez Blanco et al., 1991; Oliveira et al., 2007; Blanco \& Folegatti, 2008), arroz (Akita \& Cabuslay, 1990; Tsuchiya et al., 1992); cevada (Suhayda et al., 1992), feijão (Milia et al., 1989; Kulkarni \& Karadge, 1991; Goetz \& Coons, 1991; Assis, 1995; Sousa, 1995), e alface (Viana et al., 2004; Andriolo et al., 2005).

O feijão (Phaseolus vulgaris L.) apresenta decréscimo na produção de grãos quando cultivado em solo com salinidade no extrato de saturação maior que $1,0 \mathrm{dS} \mathrm{m}^{-1}$ a $25^{\circ} \mathrm{C}$ (Ayers \& Westcot, 1991).

O feijão apesar de reconhecido como sensível ao estresse salino, possui variedades com certa tolerância à salinidade que, uma vez identificadas, poderão ser usadas em estudos e em cultivos comerciais em áreas salinizadas. Essa identificação torna-se complexa, uma vez que o comportamento de variáveis de crescimento, geralmente usadas em estudos de seleção de espécies, é, em parte, uma resposta de adaptação aos efeitos osmótico, tóxico e nutricional do estresse salino.

O efeito osmótico do estresse salino pode ser suportado pelo ajuste osmótico de células, favorecido pela redução da área foliar e pela concentração de osmorreguladores orgânicos e inorgânicos. Quando esse ajuste se dá pelo acúmulo de íons inorgânicos e a planta suporta ou evita o efeito tóxico dos íons, a massa de matéria seca das folhas pouco será alterada, verificando-se um crescimento em espessura. Por outro lado, caso a planta não tenha mecanismos para evitar a toxidez de íons específicos, poderá ocorrer expansão celular, devido ao ajuste osmótico, e incremento da área foliar. Contudo, o efeito tóxico de íons específicos reduziria a fixação líquida de carbono (Seemann \& Critchley, 1985) e, consequentemente, a produção de massa seca. Isso sugere que massa seca e área foliar nem sempre se adequariam para separar, com segurança, genótipos quanto à tolerância à salinidade, uma vez que essas variáveis podem ser influenciadas pelo mecanismo de tolerância apresentado pela planta.

Pela importância da cultura do feijão no Brasil, especialmente para populações de baixa renda, como as da Região Nordeste, onde a incidência de solos afetados por sais é evidente, torna-se necessário selecionar variedades adaptadas a esse tipo de estresse, possibilitando seu cultivo sem sérias restrições à sua produtividade. 
Assim, neste estudo objetivou-se avaliar e selecionar variedades de feijoeiro (Phaseolus vulgaris L.) quanto à tolerância ao estresse salino e identificar, usando variedades de feijoeiro com diferentes graus de tolerância, variáveis que auxiliem na discriminação de variedades de feijoeiro quanto à tolerância a esse tipo de estresse, independentemente do mecanismo apresentado pela planta.

\section{MATERIAL E MÉTODOS}

Neste trabalho, executado em duas etapas, no Departamento de Solos da Universidade Federal de Viçosa, utilizaram-se 48 variedades de feijão, obtidas do banco de germoplasma da UFV.

\section{Seleção de variedades de feijão quanto à tolerância à salinidade}

Plântulas das variedades de feijoeiro obtidas de sementes germinadas em papel-toalha foram transferidas para as parcelas contendo as soluções nutritivas, cinco dias após a germinação, quando as folhas cotiledonares estavam completamente expandidas. As parcelas foram dispostas em blocos casualizados, com quatro repetições, e corresponderam à solução nutritiva normal (SNN) ou à solução nutritiva teste (SNT), em que a condutividade elétrica da $\operatorname{SNN}\left(0,81 \mathrm{dS} \mathrm{m}^{-1}\right)$ foi aumentada para 5,6 dS m$~^{-1}$, indicada por Araújo et al. (1992) como nível de salinidade adequado para "screen" de feijoeiros em relação à tolerância ao estresse salino. As subparcelas corresponderam a 48 variedades de feijão, provenientes do banco de germoplasma da UFV. Cada repetição correspondeu a uma planta de cada variedade, colocada juntamente numa caixa plástica contendo 30 litros de solução nutritiva ( $\mathrm{SN})$.

A SNN continha 2,0 mmol L-1 de $\mathrm{KNO}_{3}, 1,7 \mathrm{mmol} \mathrm{L}^{-1}$ de $\mathrm{Ca}\left(\mathrm{NO}_{3}\right)_{2}, 0,8 \mathrm{mmol} \mathrm{L}^{-1}$ de $\mathrm{NH}_{4} \mathrm{H}_{2} \mathrm{PO}_{4}, 0,2 \mathrm{mmol} \mathrm{L}^{-1} \mathrm{de}$ $\mathrm{MgSO}_{4}, 40 \mu \mathrm{mol} \mathrm{L}{ }^{-1}$ de Fe- EDTA, $19 \mu \mathrm{mol} \mathrm{L}^{-1} \mathrm{de}_{3} \mathrm{BO}_{3}, 7$ $\mu \mathrm{mol} \mathrm{L}-1$ de $\mathrm{MnCl}_{2}, 2 \mu \mathrm{mol} \mathrm{L}-1$ de $\mathrm{ZnSO}_{4}, 0,5 \mu \mathrm{mol} \mathrm{L}{ }^{-1}$ de $\mathrm{CuSO}_{4}$ e $0,086 \mu \mathrm{mol} \mathrm{L}^{-1} \mathrm{de}\left(\mathrm{NH}_{4}\right)_{6} \mathrm{Mo}_{7} \mathrm{O}_{24}$ (Ruiz et al., 1988). $\mathrm{O}$ pH da SN foi mantido em 5,5 \pm 0,1, com controle diário, corrigido com $\mathrm{NaOH}$ ou $\mathrm{HCl}$. A SN foi renovada a cada sete dias, para assegurar condições mais uniformes nos tratamentos. Para estimar as concentrações de cloreto de sódio necessárias para atingir as condutividades elétricas e pressões osmóticas de trabalho, prepararam-se curvas de calibração, adicionando-se à SN básica $0,20,40$, 60, 80 e $100 \mathrm{mmol} \mathrm{L}^{-1} \mathrm{de} \mathrm{NaCl}$, em três repetições. Nessas soluções, determinaram-se a condutividade elétrica (CE) e a pressão osmótica (ð), ajustando-se as seguintes equações de regressão:

$$
\begin{aligned}
& \mathrm{CE}=0,8727+0,1087 * *[\mathrm{NaCl}]-0,00018 * *[\mathrm{NaCl}]^{2} \\
& \mathrm{R}^{2}=0,999 \\
& \pi=0,03305+0,00461 * *[\mathrm{NaCl}] \quad \mathrm{R}^{2}=0,997
\end{aligned}
$$

em que $\mathrm{CE}$ é expressa em dS m ${ }^{-1}, \pi$ em $\mathrm{MPa}$ e $[\mathrm{NaCl}] \mathrm{em}$ $\mathrm{mmol} \mathrm{L} \mathrm{L}^{-1}$.

Relacionando-se a pressão osmótica e a condutividade elétrica, ajustou-se a seguinte equação: $\pi=0,02263+$ $0,05055^{* *} \mathrm{CE}$ e $\mathrm{R}^{2}=0,998$.

Vinte e um dias após a transferência das plântulas para a SN o experimento foi concluído. A parte aérea foi coletada e seca em estufa de ventilação forçada a $65^{\circ} \mathrm{C}$. Determinadas as massas de matéria seca correspondentes às plantas desenvolvidas em solução nutritiva normal (MSSNN) e aquelas sob solução nutritiva teste (MSSNT), com sais, calculou-se a relação percentual de crescimento $(\mathrm{RP}=\mathrm{MSSNT} / \mathrm{MSSNN}$ x 100), que foi utilizada no agrupamento das variedades pelo teste de Scott-Knott a $10 \%$.

As 12 variedades que se mostraram mais tolerantes foram submetidas a uma segunda avaliação, na qual aumentou-se a concentração de cloreto de sódio, elevandose a CE da SNT a 7,5 $\mathrm{dS} \mathrm{m}^{-1}$, sendo utilizado o mesmo método da primeira avaliação.

\section{Identificação de variáveis indicadoras do grau de tolerância ao estresse salino}

Dentre as 48 variedades testadas na etapa anterior, escolheram-se, com base no ranqueamento e agrupamento das médias de RP das variedades de feijoeiro pelo critério de Scott-Knott, duas variedades tolerantes (Vermelho e CNF 5547), uma medianamente tolerante (FT 83-86) e uma sensível (LM 30074), para identificar as variáveis que melhor indicassem o grau de tolerância ao estresse salino.

Este experimento foi também realizado em casa de vegetação, com tratamentos resultantes de um arranjo fatorial dessas quatro variedades, com cinco níveis de salinidade $\left(0,81 ; 2,7 ; 4,6 ; 6,5 ;\right.$ e $\left.8,4 \mathrm{dS} \mathrm{m}^{-1}\right)$, alcançados pela adição de $\mathrm{NaCl}$ à solução nutritiva específica para feijão (Ruiz et al., 1988), exceto para $0,81 \mathrm{dS} \mathrm{m}^{-1}$, que foi a condutividade elétrica normal daquela solução. O experimento, em blocos casualizados, teve quatro repetições, com duas plantas por vaso, sendo conduzido como no primeiro.

Vinte e um dias após o transplantio, finalizou-se o experimento, coletando-se as plantas. A parte aérea foi lavada rapidamente, com água desmineralizada, para remover as impurezas externas. Separaram-se caule, pecíolos e folhas, determinando-se, imediatamente, a área foliar (AF), em $\mathrm{cm}^{2}$, com um equipamento Li-Cor, modelo Li 3000. O sistema radicular também foi lavado, porém por um período de 15 minutos. Todas as partes foram secas em estufa de ventilação forçada a $65{ }^{\circ} \mathrm{C}$, até peso constante, para obtenção da massa de matéria seca $(\mathrm{mg})$. A área foliar específica (AFE) foi calculada, dividindo-se a AF pela massa de matéria seca das folhas.

$\mathrm{Na}$ análise estatística utilizou-se o programa SAEG/ UFV, comparando-se as variedades pelos contrastes ortogonais: 
$\mathrm{C} 1=$ variedade $1+$ variedade $2+$ variedade 3 - variedade 4

$\mathrm{C} 2=$ variedade $1+$ variedade 2 - variedade 3 , e C3 $=$ variedade 1 - variedade 2 .

Realizou-se análise de regressão para estudo do efeito da concentração salina sobre as respostas de massa de matéria seca de caule, pecíolos e folhas, AF e AFE, para cada variedade de feijoeiro. O critério para a escolha do modelo final de regressão foi a significância de até $10 \%$ de seus coeficientes pelo teste t. $\mathrm{Na}$ eventualidade de mais de um modelo satisfazer essa exigência, optou-se pelo de maior coeficiente de determinação ajustado.

\section{RESULTADOS E DISCUSSÃO}

A relação percentual entre a massa de matéria seca da parte aérea de plantas de feijoeiro desenvolvidas em solução nutritiva salina e em solução normal (RP) variou de 138,7 a 54,1\%. Utilizando-se o teste de Scott-Knott a 10\%, as variedades de feijoeiro foram discriminadas em duas populações: uma com 14 variedades mais "tolerantes" (RP e" 96,3\%) e outra com 34 variedades moderadamente tolerantes e sensíveis, RP d" 95,2\% (Tabela 1).

A RP de todas as variedades aqui testadas, quando crescidas em SN de salinidade igual a $5,6 \mathrm{dS} \mathrm{m}^{-1}$, foi superior à obtida por Araújo et al. (1992) em feijoeiros modera- damente tolerantes ao estresse salino, crescidos em SN com salinidade igual à indicada. Naquele trabalho, utilizando vasos isolados, a RP atingiu valor próximo a 50\%. No presente experimento, maiores valores das RP podem ser atribuídos à época diferente de plantio (período frio), que pode ter favorecido a redução da atividade química dos íons na solução e transpiração das plantas.

As variedades que apresentaram RP maior que 95\% na primeira seleção, com exceção da RAB-94 e W 22-50, por falta de material na época de instalação do experimento, quando submetidas a $\mathrm{SN}$ com 7,5 $\mathrm{dS} \mathrm{m}^{-1}$, foram ainda mais afetadas, tendo a RP variado de 59,4 a 38,3\% . As variedades Vermelho e CNF 5547 foram as que mais cresceram, e a 84 VAN 166 a que menos cresceu nessa condição (Tabela 2). Provavelmente, a menor RP observada ocorreu em razão do incremento de sal. Além disso, a tolerância manifestada pela planta não foi a mesma do experimento anterior. Isso indica que em estudos sobre mecanismos de tolerância ao estresse salino é sempre aconselhável identificar materiais sob condições, as mais semelhantes possíveis daquelas da região problema alvo, para a qual se objetiva minimizar os prejuízos e, ou, incorporá-la ao sistema produtivo. Isso acontece porque a variabilidade espacial, temporal, qualitativa e quantitativa do estresse não permite a identificação de um genótipo universalmente tolerante.

Tabela 1. Relação percentual (RP) ${ }^{1}$ entre a massa de matéria seca da parte aérea de 48 variedades de feijoeiros crescidas em solução nutritiva teste $\left(5,6 \mathrm{dS} \mathrm{m}^{-1}\right)$ e solução nutritiva normal $\left(0,81 \mathrm{dS} \mathrm{m}^{-1}\right)$

\begin{tabular}{|c|c|c|c|c|c|}
\hline Registro UFV & Variedade & $\mathbf{R P}(\%)$ & Registro UFV & Variedade & $\mathbf{R P}(\%)$ \\
\hline 2239 & ESAL 648 & $138,7 \mathrm{a}$ & 2234 & ESAL 640 & $87,1 \mathrm{~b}$ \\
\hline 896 & Ricopardo & $138,5 \mathrm{a}$ & 2135 & ESAL 565 & $87,0 \mathrm{~b}$ \\
\hline 23 & Rico 23 & $124,5 \mathrm{a}$ & 1735 & Rico 1735 & $86,1 \mathrm{~b}$ \\
\hline 2104 & Mulatinho 2104 & $117,7 \mathrm{a}$ & 2043 & LM 21135 & $84,3 \mathrm{~b}$ \\
\hline 2174 & 84 VAN 166 & $115,4 \mathrm{a}$ & 1998 & ICA Tundama & $84,2 \mathrm{~b}$ \\
\hline 2157 & RAB-94 & $111,7 \mathrm{a}$ & 2189 & WAF 16 & $83,3 \mathrm{~b}$ \\
\hline 2164 & CNF 5547 & $106,0 \mathrm{a}$ & 2135 & CNF 5548 & $82,8 \mathrm{~b}$ \\
\hline 2240 & ESAL 633 & $104,4 \mathrm{a}$ & 2048 & LM 20816 & $82,6 b$ \\
\hline 1959 & 3720 & $99,9 \mathrm{a}$ & 1958 & 3272 & $82,1 \mathrm{~b}$ \\
\hline 2163 & BZ 1185-1 & $99,5 \mathrm{a}$ & 1732 & Milionário & $81,2 b$ \\
\hline 2246 & Vermelho & $99,2 \mathrm{a}$ & 1919 & Ouro & $80,6 \mathrm{~b}$ \\
\hline 2172 & Vermelho x Milionário & $98,4 \mathrm{a}$ & 2142 & FT $83-86$ & $79,2 \mathrm{~b}$ \\
\hline 1992 & Ouro Negro & 97,9 a & 1030 & Carioca & $78,5 \mathrm{~b}$ \\
\hline 2041 & W 22-50 & $96,3 \mathrm{a}$ & 2141 & FT 120 & $77,8 \mathrm{~b}$ \\
\hline 1719 & Rio Tibagi & $95,2 \mathrm{~b}$ & 1677 & Calima & $76,2 \mathrm{~b}$ \\
\hline 2250 & Vermelho & $94,4 \mathrm{~b}$ & 2063 & LM 10089-0 & $74,4 \mathrm{~b}$ \\
\hline 2230 & PF 721218 & $92,1 \mathrm{~b}$ & 2168 & CNF 5552 & $71,5 \mathrm{~b}$ \\
\hline 2158 & AN 512706-0 & $91,2 \mathrm{~b}$ & 2173 & Rico $23=/$ TO & $71,1 \mathrm{~b}$ \\
\hline 2145 & FT 84-77 & $90,8 \mathrm{~b}$ & 2050 & LM 30030 & $68,5 \mathrm{~b}$ \\
\hline 2183 & WAF 7 & $90,6 \mathrm{~b}$ & 1923 & CNF 290 & $67,4 \mathrm{~b}$ \\
\hline 2134 & ESAL 566 & $90,5 \mathrm{~b}$ & 977 & Manteigão 977 & $66,5 \mathrm{~b}$ \\
\hline 897 & Negrito & $90,5 \mathrm{~b}$ & 2231 & ESAL 627 & $65,3 \mathrm{~b}$ \\
\hline 11 & Manteigão Fosco & $89,5 \mathrm{~b}$ & 2177 & Linhagem 2177 & $60,6 \mathrm{~b}$ \\
\hline 2136 & ESAL 563 & $87,3 \mathrm{~b}$ & 2045 & LM 30074 & $54,1 \mathrm{~b}$ \\
\hline
\end{tabular}

${ }^{1}$ Médias seguidas da mesma letra pertencem ao mesmo grupo pelo teste de Scott-Knott a $10 \%$ de probabilidade. 
Tabela 2. Relação percentual (RP) entre a massa de matéria seca da parte aérea de 12 variedades de feijoeiro crescidas em solução nutritiva teste $\left(7,5 \mathrm{dS} \mathrm{m}^{-1}\right)$ e solução nutritiva normal $\left(0,81 \mathrm{dS} \mathrm{m}^{-1}\right)$

\begin{tabular}{lcc}
\hline Registro UFV & Variedade & RP(\%) \\
\hline 2246 & Vermelho & $59,4 \mathrm{a}$ \\
2164 & CNF 5547 & $58,1 \mathrm{a}$ \\
2104 & Mulatinho 2104 & $53,3 \mathrm{a}$ \\
3720 & 3720 & $52,5 \mathrm{a}$ \\
2172 & Vermelho x Milionário & $51,4 \mathrm{a}$ \\
23 & Rico 23 & $51,0 \mathrm{a}$ \\
2239 & ESAL 648 & $47,4 \mathrm{a}$ \\
2240 & ESAL 633 & $46,8 \mathrm{a}$ \\
2163 & BZ 1185-1 & $44,4 \mathrm{a}$ \\
896 & Ricopardo & $44,0 \mathrm{a}$ \\
1992 & Ouro Negro & $43,2 \mathrm{a}$ \\
2174 & 84 VAN 166 & $38,3 \mathrm{a}$ \\
\hline
\end{tabular}

${ }^{1}$ Médias seguidas da mesma letra pertencem ao mesmo grupo pelo teste de Scott-Knott a $10 \%$ de probabilidade.

A interação entre os fatores variedades e níveis de salinidade foi significativa para todas as variáveis analisadas, observando-se que as variáveis de crescimento foram significativamente modificadas pela salinidade da $\mathrm{SN}$, sendo menos afetadas no grupo tolerante, diferenciando-se, estatisticamente, daquelas da LM 30074 (Tabela 3). A massa de matéria seca seca das raízes da LM 30074 cresceu até $3,7 \mathrm{dS} \mathrm{m}^{-1}$, porém decresceu bruscamente a partir deste nível de salinidade. No caule, nos pecíolos e nas folhas, entretanto, a produção de massa de matéria seca da LM 30074 começou a diminuir já a partir de 2,0, 1,3 e $1,5 \mathrm{dS} \mathrm{m}^{-1}$, respectivamente, mas numa taxa menor que nas raízes, pelo menos até aproximadamente $4,6 \mathrm{dS} \mathrm{m}^{-1}$. A redução na produção de massa de matéria seca da CNF 5574 foi semelhante à da LM 30074, pelo menos na massa seca de raiz e de folha. Na Vermelho e FT 83-86, nas diversas partes das plantas, a produção da massa de matéria seca decresceu, linearmente, com o aumento da salinidade. No entanto, as plantas foram menos afetadas que a LM 30074, principalmente quando o estresse salino ultrapassou $4,6 \mathrm{dS} \mathrm{m}^{-1}$ (Tabela 3 ).

$\mathrm{A}$ área foliar das quatro variedades avaliadas decresceu com o incremento da salinidade das soluções (Tabela 3). A redução da área foliar, provavelmente, concorre com a diminuição do volume das células. $\mathrm{O}$ aumento da espessura das folhas da LM 30074, observado visualmente, porém, parece ter contribuído para que a produção de massa de matéria seca tivesse sido menos afetada até, aproximadamente, 4,6 $\mathrm{dS} \mathrm{m}{ }^{-1}$. Acentuada redução da área foliar de feijoeiros cultivados sob estresse salino, em solução nutritiva, foi, também, observada por Wignarajah et al. (1975) e atribuída à redução do tamanho das células do parênquima paliçádico. Esses autores verificaram, ainda, aumento significativo da espessura das folhas em consequência do aumento da espessura das células do parênquima lacunoso.
A redução da área foliar, por reduzir o volume das células, pode ter contribuído para o ajuste osmótico das células, admitindo-se que a quantidade de soluto absorvido foi concentrada em menor volume de suco celular, conforme já sugerido por Greenway \& Munns (1980). Resultados semelhantes foram obtidos por Sanchez Blanco et al. (1991) que, em tomate, encontraram maior redução da área foliar que da massa de matéria seca produzida em altos níveis de salinidade, apesar da turgescência das folhas ter sido mantida.

A produção de massa de matéria seca da FT 83-86 foi, estatisticamente, igual à da Vermelho e CNF 5574, exceto quanto à produção de massa de matéria seca do caule (Tabela 3). Isso indica que a variável usada no screen (relação percentual de crescimento com base na produção de massa de matéria seca das partes aérea e total) pode não ser um indicador seguro da sensibilidade ao estresse salino. Percebe-se ainda na Tabela 3 que apenas a área foliar revelou diferença entre Vermelho e CNF 5574 (tolerantes), indicando maior eficiência na discriminação de tolerância de feijoeiros aos sais, principalmente quando se compararem variedades tolerantes.

A variedade sensível LM 30074 apresentou menor produção de massa de matéria seca da parte aérea do que as tolerantes em todo o intervalo experimental de salinidade, o que só ocorreu para área foliar com salinidade superior a $5,8 \mathrm{dS} \mathrm{m}^{-1}$, permitindo se aventar que a massa de matéria seca e a área foliar, tomadas isoladamente, não sejam indicadores confiáveis da sensibilidade de feijoeiros à salinidade, contrariamente ao sugerido por Araújo et al. (1992). Estes autores trabalharam com uma variedade cuja sensibilidade foi atribuída à exclusão de $\mathrm{Na}$ e $\mathrm{K}$ da parte aérea. Assim, o decréscimo da área foliar não contribuiu para o ajuste osmótico e indicou bem a sensibilidade daquela variedade.

O efeito da salinidade sobre a massa de matéria seca das raízes, da parte aérea e sobre a área foliar foi consistente com os resultados obtidos em outros estudos, nos quais o excesso de $\mathrm{NaCl}$ nos substratos reduziu o crescimento e o desenvolvimento dessas variáveis em várias espécies de interesse agronômico (Lunin et al., 1961; LaHaye \& Epstein, 1971; Greenway \& Munns, 1980; Cramer \& Läuchli, 1986; Zaffaroni et al., 1988; Salim, 1989; Akhavan-Kharazian et al., 1990; Andriolo et al., 2005; Lima \& Bull, 2008).

Aárea foliar específica (AFE) em Vermelho, CNF 5574 e FT 83-86 não variou com a salinidade, enquanto, na LM 30074 decresceu até uma salinidade igual a 2,92 dS m${ }^{-1} \mathrm{e}$ aumentou até o nível mais elevado de salinidade testado (Figura 1). Como se verificou aumento de massa de matéria seca nas folhas de LM 30074 até $1,5 \mathrm{dS} \mathrm{m}^{-1}$, admite-se que, nessa faixa de salinidade, a menor AFE indicaria aumento líquido na produção de massa de matéria seca, 
Tabela 3. Valores médios da produção de massa de matéria seca (mg) das raízes, do caule, dos pecíolos, das folhas, da parte aérea e da área foliar $\left(\mathrm{cm}^{2}\right)$ em quatro variedades de feijoeiro (Vermelho, CNF 5574, FT 83-86 e LM 30074), em diferentes condutividades elétricas da solução de crescimento (CE); e contrastes ortogonais comparando as variedades de feijão

\begin{tabular}{|c|c|c|c|c|c|c|c|}
\hline \multirow{3}{*}{ Variedade } & \multirow{3}{*}{$\begin{array}{c}\mathrm{CE} \\
\left(\mathrm{dS} \mathbf{m}^{-1}\right)\end{array}$} & \multicolumn{5}{|c|}{ Produção de matéria seca } & \multirow{3}{*}{$\begin{array}{l}\text { Área foliar } \\
\qquad\left(\mathbf{c m}^{2}\right)\end{array}$} \\
\hline & & Raízes & Caule & Pecíolos & Folhas & Parte aérea & \\
\hline & & \multicolumn{5}{|c|}{ (mg) } & \\
\hline \multirow{5}{*}{ V1-Vermelho } & 0,81 & 519 & 702 & 229 & 1.727 & 2.658 & 737,21 \\
\hline & 2,70 & 464 & 552 & 178 & 1.208 & 1.938 & 627,35 \\
\hline & 4,60 & 484 & 502 & 154 & 1.303 & 1.959 & 655,41 \\
\hline & 6,50 & 383 & 424 & 162 & 976 & 1.562 & 496,72 \\
\hline & 8,40 & 306 & 250 & 102 & 626 & 979 & 365,16 \\
\hline \multirow[t]{5}{*}{ V2-CNF 5547} & 0,81 & 540 & 663 & 258 & 1.533 & 2.453 & 620,32 \\
\hline & 2,70 & 604 & 616 & 236 & 1.240 & 2.091 & 575,67 \\
\hline & 4,60 & 618 & 499 & 227 & 1.110 & 1.835 & 449,18 \\
\hline & 6,50 & 532 & 381 & 153 & 849 & 1.383 & 384,72 \\
\hline & 8,40 & 205 & 144 & 68 & 329 & 541 & 250,14 \\
\hline \multirow[t]{5}{*}{ V3-FT 83-86 } & 0,81 & 603 & 579 & 278 & 1.697 & 2.555 & 556,96 \\
\hline & 2,70 & 542 & 497 & 211 & 1.523 & 2.231 & 795,47 \\
\hline & 4,60 & 540 & 393 & 228 & 1.227 & 1.849 & 476,47 \\
\hline & 6,50 & 461 & 345 & 160 & 969 & 1.474 & 457,94 \\
\hline & 8,40 & 310 & 202 & 101 & 663 & 965 & 333,31 \\
\hline \multirow[t]{5}{*}{ V4-LM 30074} & 0,81 & 405 & 363 & 212 & 1.416 & 1.991 & 629,28 \\
\hline & 2,70 & 445 & 324 & 194 & 1.287 & 1.805 & 550,74 \\
\hline & 4,60 & 502 & 294 & 162 & 1.011 & 1.467 & 413,89 \\
\hline & 6,50 & 465 & 255 & 128 & 947 & 1.331 & 298,12 \\
\hline & 8,40 & 37 & 41 & 22 & 63 & 127 & 148,69 \\
\hline \multicolumn{8}{|c|}{ Contrastes ortogonais } \\
\hline \multicolumn{2}{|l|}{$\mathrm{V} 1+\mathrm{V} 2+\mathrm{V} 3$ vs $\mathrm{V} 4$} & $309,8 * *$ & $583,6^{* *}$ & $118,2 * *$ & $561,6 * *$ & $1.260,0 * *$ & $332,0 *$ \\
\hline \multicolumn{2}{|l|}{$\mathrm{V} 1+\mathrm{V} 2$ vs V3 } & $-51,4 \mathrm{~ns}$ & $104,2^{*}$ & $-37,8 \mathrm{~ns}$ & $-251,4 \mathrm{~ns}$ & $-149,8 \mathrm{~ns}$ & $-15,7 \mathrm{~ns}$ \\
\hline \multicolumn{2}{|l|}{ V1 vs V2 } & $-68,6 \mathrm{~ns}$ & $25,4 \mathrm{~ns}$ & $-23,4 \mathrm{~ns}$ & $155,8 \mathrm{~ns}$ & $158,6 \mathrm{~ns}$ & $120,4^{*}$ \\
\hline
\end{tabular}

*, ** Significativo a 5 e $1 \%$, respectivamente, pelo teste $\mathrm{F}$; ns - Não significativo.

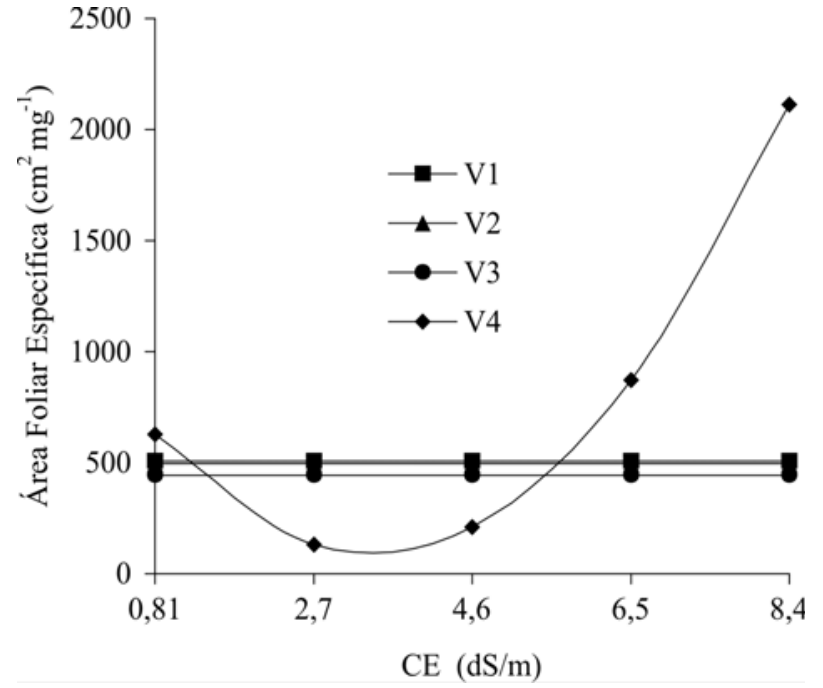

Figura 1. Área foliar específica (AFE) em quatro variedades de feijoeiro (V1-Vermelho, V2-CNF 5574, V3-FT 83-86 e V4-LM 30074) em função da condutividade elétrica da solução de crescimento (CE). notadamente devido ao aumento da espessura das células, ocorrido por um possível ajuste osmótico, resultante da concentração de solutos, contribuindo para expansão celular e manutenção do turgor (Sanchez Blanco et al., 1991; Mahmood \& Quarric, 1993).

Esse resultado também pode ser justificado admitindo-se a possibilidade de ocorrência de um ajustamento mecânico devido ao aumento da extensibilidade da parede celular, contrabalanceando a redução do turgor celular, mantendo a taxa de crescimento da folha, como verificado por Newmann et al. (1988) em plântulas de Phaseolus vulgaris $\mathrm{L}$. tratadas com $\mathrm{NaCl}$.

O fato de ter sido verificado em LM 30074, o incremento na massa de matéria seca das folhas e a redução da AFE permite descartar, com certa segurança, a hipótese de que tenha ocorrido significativa limitação na absorção líquida de $\mathrm{CO} 2$, causada pelo excesso de sais, pelo menos até uma CE de $1,5 \mathrm{dS} \mathrm{m}^{-1}$. No entanto, nos níveis mais elevados de salinidade (acima de $1,5 \mathrm{dS} \mathrm{m}^{-1}$ ) 
o aumento da AFE e a queda da massa de matéria seca produzida indicam redução da incorporação líquida de $\mathrm{CO} 2$, possivelmente devido aos efeitos nutricionais e, ou, tóxicos. O aumento da AFE demonstra expansão celular, donde se aventou que plantas da variedade sensível (LM 30074) não tenham sofrido limitação de água, resistindo assim ao efeito osmótico. Resultados como esses sugerem que se deve procurar identificar qual dos componentes do estresse salino (osmótico ou nutricional) afeta mais a cultura que está sendo avaliada. Isso pode ser alcançado para feijão, determinando-se o teor de sódio nas folhas e a área foliar específica (Araújo et al., 1994).

\section{CONCLUSÕES}

As variedades de feijoeiro do banco de germoplasma da UFV avaliadas apresentam variabilidade genética para a tolerância ao estresse salino, indicando a possibilidade de se praticar seleção e melhoramento para o caráter.

Foram identificados dois grupos: variedades tolerantes: Vermelho, CNF 5547, Mulatinho 2104, 3720, Vermelho x Milionário, Rico 23, ESAL 648, ESAL 633, BZ 1185-1, Ricopardo, Ouro Negro, 84 VAN 166, RAB 94 e W 22-50; e variedades sensíveis: Rio Tibagi, Vermelho, PF 721218, AN 512706-0, FT 84-77, WAF 7, ESAL 566, Negrito, Manteigão Fosco, ESAL 563, ESAL 640, ESAL 565, Rico 1735, LM 21135, ICA Tundama, WAF 16, CNF 5548, LM 20816, 3272, Milionário, Ouro, FT 83-86, Carioca, FT 120, Calima, LM 10089-0, CNF 5552, Rico 23 - TO, LM 30030, CNF 290, Manteigão 977, ESAL 627, Linhagem 2177 e LM 30074.

Comparativamente a outras variáveis de crescimento, a área foliar específica é o melhor índice para discriminar feijoeiros quanto à tolerância à salinidade, independentemente dos mecanismos de respostas ao estresse salino por eles apresentados.

\section{REFERÊNCIAS}

Akhavan-Kharazian M, Campbell WF, Jurinak JJ \& Dud Ley LM (1990) Calcium amelioration of $\mathrm{NaCl}$ on plant growth, chlorophyll, and ion concentration in Phaseolus vulgaris. Arid Soil Research Rehabilitation, 5:9-19.

Akita S \& Cabuslay GS (1990) Physiological basis of differential response to salinity in rice cultivars. Plant and Soil, 123:277294.

Allison LE (1964) Salinity in relation to irrigation. Advances in Agronomy, 16:139-178.

Andriolo JL, Luz GL, Witter MH, Godoi RS, Barros GT \& Bortolotto OC (2005) Growth and yield of lettuce plants under salinity. Horticultura Brasileira, 23:931-934.

Araújo CAS, Nunes JC \& Ruiz HA (1992) Resposta de quatro linhagens de feijão a doses de cloreto de sódio. In: Reunião Brasileira de Manejo e Conservação do Solo e da Água, Jaboticabal. Anais, SBCS/UNESP/SCTDE, p.185.
Araújo CAS, Ruiz HÁ \& Neves JCL (1994) Uso da área foliar específica e do teor de sódio na identificação dos componentes do "stress" salino mais prejudiciais ao feijoeiro. In: Reunião Brasileira de Fertilidade do Solo e Nutrição de Plantas, Petrolina. Anais, SBCS/EMBRAPA-CPATSA, p.213-215.

Assis JS (1995) Efeito do estresse salino sobre o metabolismo do nitrogênio, o crescimento e a produção de dois cultivares de feijão (Phaseolus vulgaris L.). Tese de Doutorado. Botucatu, UNESP. 97p.

Ayers RS \& Westcot DA (1991) A qualidade da água na agricultura. Roma, FAO Irrigação e Drenagem, 29 Revisado. 218p.

Blanco FF \& Folegatti MV (2008) Doses de N e K no tomateiro sob estresse salino: II. Crescimento e partição de matéria seca. Revista Brasileira de Engenharia Agrícola e Ambiental, 12:3440 .

Chartzoulakis KS (1991) Effects of saline irrigation water on germination, growth and yield of greenhouse cucumber. Acta Horticulturae, 287:327-334.

Cramer GR \& Läuchli A (1986) Ion activities in solution in relation o $\mathrm{Na}+-\mathrm{Ca} 2+$ interaction at the plasmalemma. Journal of Experimental Botany, 37:321-330.

Epstein E \& Rains DW (1987) Advances in salt tolerance. Plant and Soil, 99:17-29.

Fageria NK (1985) Salt tolerance of rice cultivars. Plant and Soil, $88: 237-243$.

Franco JA, Esteban C \& Rodriguez C (1993) Effects of salinity on various growth stages of muskmelon cv. Revigal. Journal of Horticultural Science, 68:899-904.

Goetz SH \& Coons JM (1991) Tolerance of tepary and navy beans to $\mathrm{NaCl}$ during germination and emergence. HortScience, 26:246-249

Greenway H \& Munns R (1980) Mechanism of salt tolerance in nonhalophytes. Annual Review of Plant Physiology, 31:149190 .

Kulkarni H \& Karadge BA (1991) Growth and mineral nutrition of moth bean (Phaseolus aconitifolius Jacq.) under saline conditions. Indian Journal of Plant Physiology, 34:14-24.

LaHaye PA \& Epstein E (1971) Calcium and salt toleration by bean plants. Physiologia Plantarum, 25:213-218.

Lima MDB \& Bull LT (2008) Produção de cebola em solo salinizado. Revista Brasileira de Engenharia Agrícola e Ambiental, 12:231235 .

Lunin J, Gallatin MH \& Batchelder AR (1961) Effect of stage of growth at the time of salinization on growth and chemical composition of beans: I. Total salinization accomplished in one irrigation. Soil Science, 91:194-203.

Mahmood A \& Quarric SA (1993) Effects of salinity on growth, ionic relations and physiological traits of wheat, disomic addition lines from Thinopyrum bessarabicum, and two amphiploids. Plant Breeding, 110:265-276.

Milia M, Murtas A \& Rivoira G (1989) Agronomic productivity at increasing salinity levels. I. Effects of salinity on biological and yield performance of two Phaseolus vulgaris L. cultivars. Rivista di Agricoltura Subtropicale e Tropicale, 83:161-172.

Newmann PM, Volkenburgh EV. \& Cleland RE (1988) Salinity stress inhibits bean leaves expansion by reducing tugor, not cell extensibility. Plant Physiology, 88:233-237.

Oliveira BC, Cardoso MAA, Oliveira JC de, Oliveira FA de \& Cavalcante LF (2007) Características produtivas do tomateiro submetido a diferentes níveis de sais, na água de irrigação. Revista Brasileira de Engenharia Agrícola e Ambiental, 11:11-16. 
Ruiz HÁ, Herkenhoff Filho HE \& Bunicenha JM (1988) Proporções e concentrações de macronutrientes na formulação de soluções nutritivas específicas para culturas de interesse agronômico. V. Feijão. In: Reunião Brasileira de Fertilidade do Solo, Guarapari. Anais, SEAG-ES/EMCAPA/EMATER-ES/SBCS, p. 120 .

Salim M (1989) Effects of $\mathrm{NaCl}$ and $\mathrm{KCl}$ salinity on growth and ionic relations of red kidney beans (Phaseolus vugaris L.). Journal Agronomic Crop Science, 163:338-344.

Sanchez Blanco MJ, Bolarin MC, Alarcon JJ \& Torrecillas A (1991) Salinity effects on water relations in Lycopersicon esculentum and its wild salt-tolerant relative species L. pennellii. Physiologia Plantarum, 83:269-274

Seemann JR \& Critchley C (1985) Effects of salt stress on the growth, ion content, stomatal behaviour and photosynthetic capacity of a salt sensitive species, Phaseolus vulgaris L. Planta, 164:151-161.

Silva MCC, Medeiros JF, Negreiros MZ \& Souza VF (2005) Produtividade de frutos do meloeiro sob diferentes níveis de salinidade da água de irrigação, com e sem cobertura do solo. Horticultura Brasileira, 23:202-205.
Sousa MR (1995) Comportamento do feijoeiro (Phaseolus vulgaris L. cv Eriparza) submetido a diferentes níveis de salinidade da água de irrigação. Tese de Mestrado. Lavras, Universidade Federal de Lavras. 94p.

Suhayda CG, Redmann RE, Harvey BL \& Cipywnyk AL (1992) Comparative response of cultivated and wild barley species to salinity stress and calcium supply. Crop Science, 32:154-163.

Tsuchiya M, Naito H, Ehara H \& Ogo T (1992) The physiological response of rice to salinity. I. The relationship between $\mathrm{Na}^{+}$ uptake and transpiration under different humidity and salinity conditions. Japanese Journal of Crop Science, 61:16-21.

Viana SBA, Fernandes PD, Gheyi HR, Soares FAL \& Carneiro PT (2004) Índices morfofisiológicos e de produção de alface sob estresse salino. Revista Brasileira de Engenharia Agrícola e Ambiental, 8:23-30.

Wignarajah K, Jennings DH \& Handley JF (1975) The effect of salinity on growth of Phaseolus vulgaris L. I. Anatomical changes in the first trifoliate leaf. Annual Botany, 39:1029-1038.

Zaffaroni E, Cavalcante LF, Araújo CAS, Militão Neto V \& Souza Jr VE (1988) Efeito da salinidade e do déficit hídrico em três genótipos de girassol. Tecnologia e Ciência, 2:203-208. 